九州大学学術情報リポジトリ

Kyushu University Institutional Repository

The Effect of the Ratio Difference of Overlapped Areas of Stereoscopic Images on Each Eye in a Teleoperation

Yamamoto, Tomohide

Department of Intelligent Systems, Graduate School of Information Science and Electrical

Engineering, Kyushu University : Graduate Student

Matsunaga, Katsuya

Department of Intelligent Systems, Faculty of Information Science and Electrical Engineering, Kyushu University

Shidoji, Kazunori

Department of Intelligent Systems, Faculty of Information Science and Electrical Engineering, Kyushu University

https://doi.org/10.15017/1515710

出版情報: 九州大学大学院システム情報科学紀要. 6 (1)，pp.13-18，2001-03-26. 九州大学大学院シス テム情報科学研究院

バージョン：

権利関係 : 


\title{
The Effect of the Ratio Difference of Overlapped Areas of Stereoscopic Images on Each Eye in a Teleoperation
}

\author{
Tomohide YAMAMOTO* , Katsuya MATSUNAGA** and Kazunori SHIDOJI**
}

(Received December 15, 2000)

\begin{abstract}
It is reported that the efficiency of a teleoperation in stereoscopic images of the working site is lower than that in the direct viewing of the site. It is assumed that one of the causes of lower efficiency of the teleoperation in the stereoscopic images is the difficulty in the fusion of images, which is caused by the imperfect overlapping of images on each eye. In most teleoperations, the convergence of the stereoscopic cameras is fixed at a certain point, usually in the middle of the working area. When the plane of the operator's eye-fixation-point is apart from the plane of the convergence point of the stereoscopic cameras, the two images do not perfectly overlap. It requires a great deal of effort for the images to be fused when the difference in the depth of the two planes is over a certain value. We hypothesized that imperfect overlapping of the images on the left and right eyes would cause a decrease in efficiency for a teleoperation. The results of the experiment led to the conclusion that in order to achieve a good performance in a teleoperation, the convergence point of the cameras should follow the target object on which subjects fixate both of their eyes.
\end{abstract}

Keywords: Stereoscopic cameras, Teleoperation, Overlapped area, Convergence, Fixation point

\section{Introduction}

There are many hazardous tasks in human life at disaster sites such as fires, space exploration and so on. In order to avoid harm to workers in such situations, work could be done using a teleoperation robot. The operation of telerobots from a remote place requires three-dimensional information about the space in which the robot works. The operators need to obtain depth cues to reconstruct the three-dimensional space. The depth cue of binocular disparity gives the strongest possibility of reconstructing the three-dimensional space within the range of around $20 \mathrm{~m}$ in other depth cues ${ }^{1)}$. It is known that a stereoscopic video system helps operators perform teleoperation faster and with less error than with a monoscopic system ${ }^{2), 3)}$. But, it was reported that the task time of the teleoperation in the conventional stereoscopic video system is longer than that of the direct view, and subjects felt more fatigued in the use of stereoscopic video images than in the direct view ${ }^{4)}$. In order to obtain high performance and less fatigue, it is proposed that a stereoscopic video system should have a wide-angle and high-resolution images ${ }^{5}$. Human eyes converge to see near objects and diverge to see far objects. In many telepresence systems, the two

* Department of Intelligent Systems,Graduate Student

** Department of Intelligent Systems video cameras for the stereoscopic cameras, are set at fixed convergence angles. Therefore, when there is a large difference in the distances between the convergence point of the cameras and the working area, the ratio of the overlapped area of the two images, which were projected to each eye, decreases. In pilot studies, it was found that the larger the ratio of the overlapped area of the two images decreased, the more difficult the fusion of the two images becomes. This may be a factor in the decline in the performance of remote control task and the cause of fatigue while observing the stereoscopic pictures. In the last experiment, subjects were required to insert a cylinder into a hole. The result was that the completion times of the operation under nearly-perfectly overlapped conditions were significantly smaller than the ones under the conditions in which the overlapping is less than the maximum value ${ }^{6)}$. However, we didn't consider the process in which subjects caught a cylinder. In actual operation, it is a very important process to catch an object when the operator moves an object and carry it. We considered the process in which subjects caught a cylinder in this research. The ratio of the overlapped area has a close relation to the camera's convergence point and the operator's fixation point. The camera's convergence point is the intersection of the optical axes of the two cameras. In Fig. 1, when the operator fixates on object $\mathrm{M}$ with both eyes, and the convergence point of the two cameras 
is set at the object $M$, the rate of the overlapped area of the images on the left and right eyes is nearly at a maximum. The rate of the overlapped area declines when the plane of the convergence point of the camera moves farther away from the plane of the point of the object $M$ while subjects fixate on it.

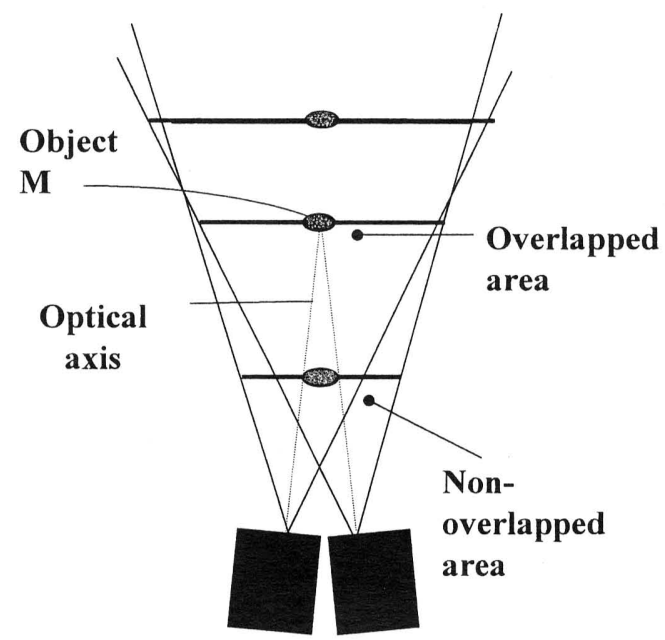

Cameras

Fig.1 Relationship between the overlapped ratio and the convergence point of cameras.

\section{Experiment}

\subsection{Purpose}

We examined the effect of the ratio difference in overlapped areas of stereoscopic images on each eye to the completion times and errors in a teleoperation.

\subsection{Method}

\subsubsection{Subject}

The subjects were 6 males whose ages ranged from 21 to 26 years. The average age was 23 years. All of the subjects had normal stereoscopic vision.

\subsubsection{Experimental system}

(Hardware): The experimental system was constructed using a workstation (Silicon Graphics: sgi540; Xeon; 500MHz) with a 17 inch CRT display, and a three-dimensional mouse with six degrees of freedom (Spacetec IMC: SPACE BALL 3003). The virtual reality space of the experiment was displayed on the 17 inch CRT. The subjects moved the gripper in the virtual reality space by using the threedimensional mouse. A liquid crystal shutter glass system (StereoGraphics: Crystal Eyes) was used to display the stereoscopic video pairs. The SyncDoubling-Emitter sends synchronous signals to liquid crystal shutters (Fig. 2).

(Software): The software was developed for this experiment by using Visual $\mathrm{C}++6.0$ and Graphic Library (Sense 8: World Tool Kit (WTK)). It was run on a workstation with Windows NT 4.0 (Fig. 3).

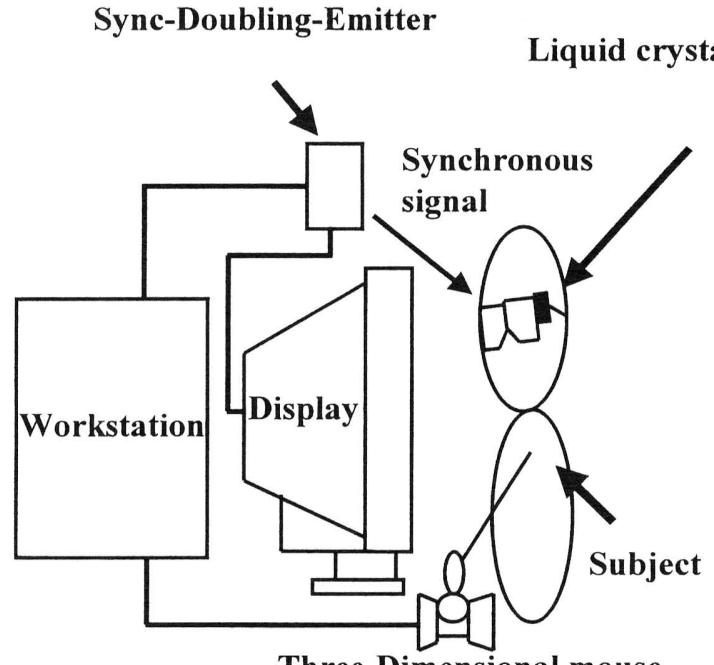

Three-Dimensional mouse

Fig.2 Block diagram of experimental system.

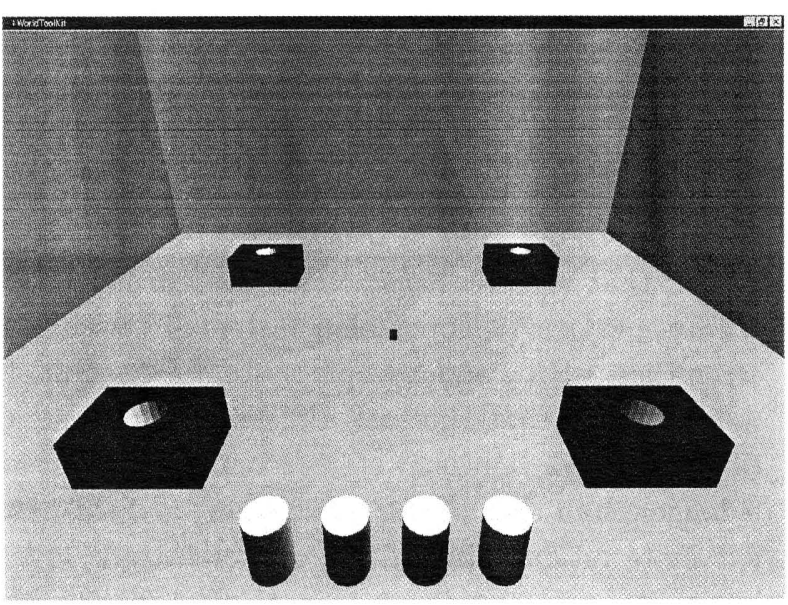

Fig.3 Virtual reality space on display.

\subsubsection{Virtual reality space}

In this experiment, we constructed a virtual reality space. We simulated the working area which size was $90 \mathrm{~cm}$ width, $90 \mathrm{~cm}$ vertical width, and $70 \mathrm{~cm}$ height in actual space. The size of the cylinders 
was $3.0 \mathrm{~cm}$ in diameter and $5.0 \mathrm{~cm}$ in height (Fig.4). The size of the gripper was $1.0 \mathrm{~cm}$ in width, $1.0 \mathrm{~cm}$ in vertical width, and $1.0 \mathrm{~cm}$ in height (Fig.5). The size of the hole-base was $10.0 \mathrm{~cm}$ in width, $7.5 \mathrm{~cm}$ in vertical width, and $5.0 \mathrm{~cm}$ in height (Fig.6). The subjects caught a cylinder using the gripper. The diameter of the hole was $3.3 \mathrm{~cm}$ and the depth was $5.0 \mathrm{~cm}$. The stereoscopic cameras were placed 70 $\mathrm{cm}$ away from the center of the working area. The distance of the two cameras was $6.5 \mathrm{~cm}$. The gripper was placed at the center of the working area. Each of the hole-bases was placed about $28 \mathrm{~cm}$ apart from the center of the working area. The angle of depression of the cameras was 26.5 degrees. The lens angle was 80 degrees.

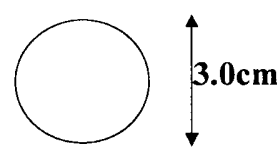

Top view

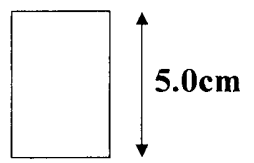

Side view
Fig.4 Figure of the cylinder.

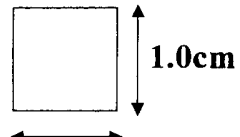

$1.0 \mathrm{~cm}$

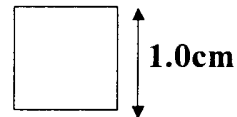

Side view
Fig.5 Figure of the gripper

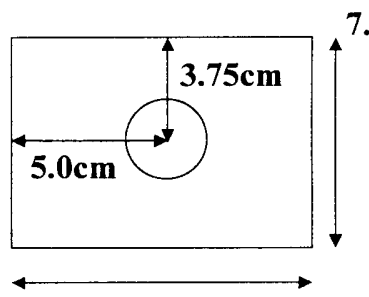

$10.0 \mathrm{~cm}$ $7.5 \mathrm{~cm}$

Top view

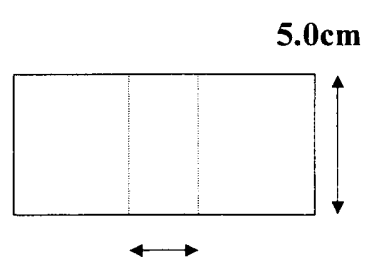

$2.4 \mathrm{~cm}$

Side view

Fig.6 Figure of the hole-base.

\subsubsection{Experimental procedure}

The subjects were required to insert a cylinder into a hole. We first instructed subjects on how to operate the three-dimensional mouse and then asked whether they could look at a stereoscopic image. They were then asked to practice moving and inserting a target object into a hole successfully prior to the actual measurements. Finally, the subjects were required to perform the task with three different camera convergence conditions in the virtual reality environment.

(Condition 1): The convergence point of the two cameras was set on the gripper, which subjects moved. When the convergence point of the cameras followed the point on the gripper, the rate of the overlapped area of the two images was nearly always at a maximum while subjects fixed their two eyes on it (Fig.7). When the gripper caught a cylinder which was the target, the convergence point of the stereoscopic cameras was set at the plane of the cylinder.
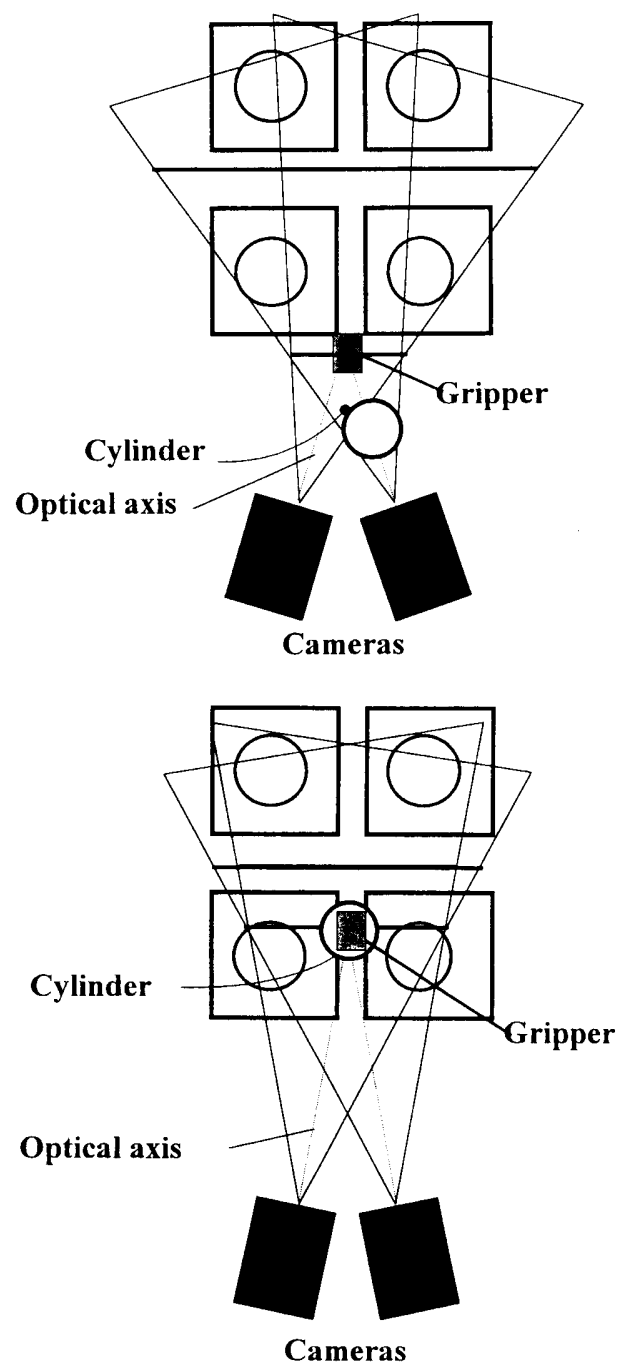

Fig.7 Experimental Condition 1

The convergence point of both cameras followed the point on the gripper.

(Condition 2): The convergence point of both cameras was not set on the gripper, but at the center of the working area. The larger the difference between the plane of the camera's convergence point and the plane of the subject's fixation point became, the more the rate of the overlapped area of the two images decreased (Fig.8). 


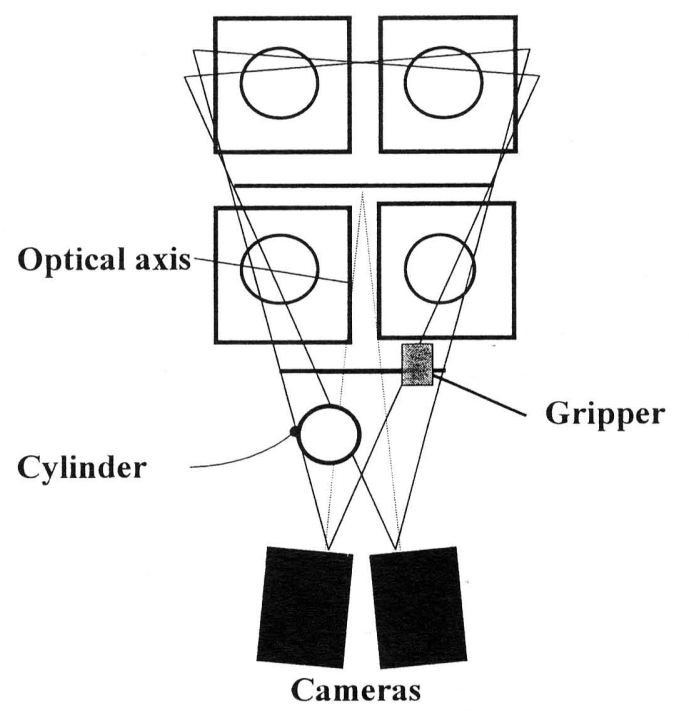

Fig.8 Experimental Condition 2

The convergence point of both cameras was set at the center of the working area.

(Condition 3): The convergence point of both cameras was not set on the gripper, but at the hole of the hole-base. The ratio of the overlapped area of the two images at the hole was nearly always at a maximum when the convergence point of the two cameras was set on the hole of the hole-base (Fig.9). The convergence point of the stereoscopic cameras was set on the hole of the hole-base, which was the next target when the subject inserts a cylinder into the hole of the hole-base.

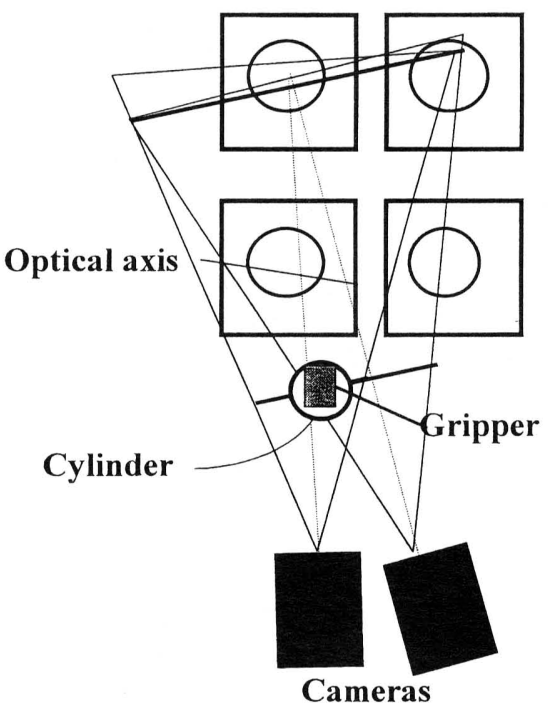

Fig.9 Experimental Condition 3

The convergence point of both cameras was set at the hole of the hole-base.

\section{Result}

The mean completion times of the task by all the subjects in Conditions 1, 2 and 3 are shown in Fig.10. The mean numbers of errors in the three conditions are shown in Fig.11. An analysis of variance $[3$ (condition) x 10 (trial)] was conducted. It was found that the completion times at Condition 1 and Condition 3 were significantly shorter than those at Condition $2(\mathrm{~F}(2,5)=5.711$,pi.05; see Table1). Concerning the mean numbers of errors, there were no significant differences in the three conditions $(\mathrm{F}(2,5)=3.776, \mathrm{n} . \mathrm{s}$; see Table2).

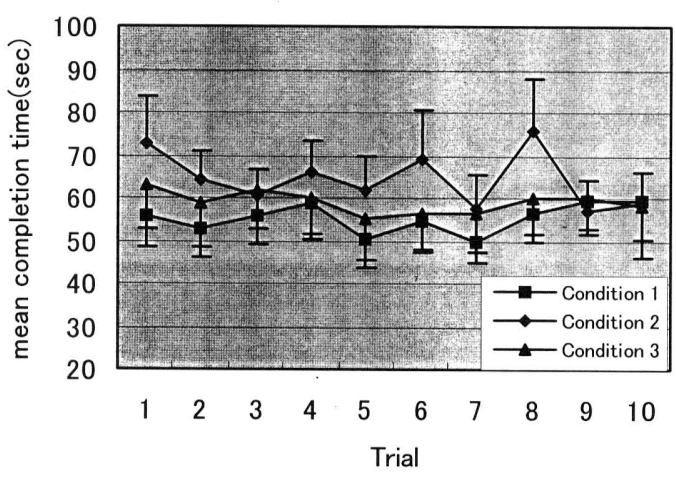

Fig.10 The mean completion times of the task by all subjects.

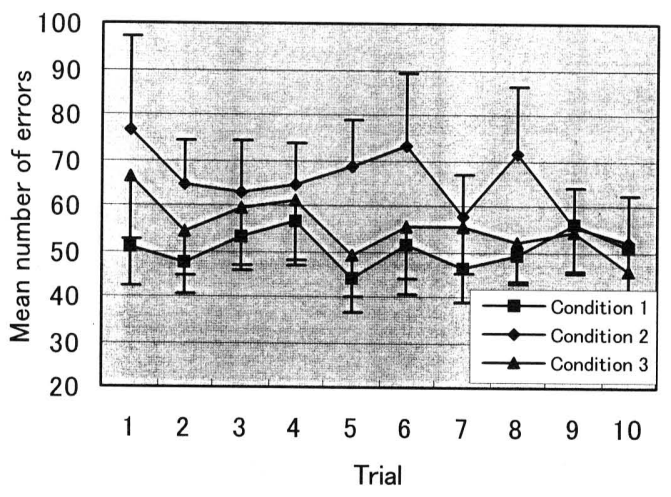

Fig.11 The mean number of errors of the task by all subjects. 
The Effect of the Ratio Difference of Overlapped Areas of Stereoscopic Images on Each Eye in a Teleoperation -17-

Table 1 Analysis of variance of the mean completion time of the task (simple main effect).

\begin{tabular}{|c|c|c|c|c|c|}
\hline Source & SS & df & MS & $\mathrm{F}$ & $\mathrm{P}(+p<.10, * p<.05)$ \\
\hline Subject & 29502.7777778 & 5 & 5900.555556 & & \\
\hline A:convergence & 6146.0777778 & 2 & 3073.03888889 & 5.711 & $0.0222^{*}$ \\
\hline Error[AS] & 5380.8555556 & 10 & 538.0855556 & & \\
\hline B:trial & 2098.7777778 & 9 & 233.1975309 & 2.124 & $0.0470^{*}$ \\
\hline Error[BS] & 4939.8888889 & 45 & 109.7753086 & & \\
\hline AB & 2096.5888889 & 18 & 116.4771605 & 1.559 & $0.0886+$ \\
\hline Error[ABS] & 6723.1444444 & 90 & 74.7016049 & & \\
\hline Total & 56888.1111111 & 179 & & & \\
\hline
\end{tabular}

Table 2 Analysis of variance of the mean number of errors (simple main effect).

\begin{tabular}{|c|c|c|c|c|c|}
\hline Source & SS & df & MS & $\mathrm{F}$ & $\mathrm{P}(+p<.10, * p<.05)$ \\
\hline Subject & 45874.8500000 & 5 & 9174.9700000 & & \\
\hline A:convergence & 10862.633333 & 2 & 5431.3166667 & 3.776 & $0.0600+$ \\
\hline Error[AS] & 14382.9666667 & 10 & 1438.2966667 & & \\
\hline B:trial & 4293.3833333 & 9 & 477.0425926 & 1.917 & $0.0737+$ \\
\hline Error[BS] & 11198.3166667 & 45 & 248.8514815 & & \\
\hline AB & 4224.7000000 & 18 & 234.7055556 & 1.226 & 0.2582 \\
\hline Error[ABS] & 17227.7000000 & 90 & 191.4188889 & & \\
\hline Total & 108064.5500000 & 179 & & & \\
\hline
\end{tabular}

\section{Discussion}

When the convergence point of the cameras follows the point on the target object on which subjects fixate both their eyes, the rate of the overlapped area of the two images is nearly always at a maximum. It was ascertained that work by a teleoperation could be done in a shorter amount of time by making the overlapping area larger. Furthermore, it was found that the work could be done more precisely. When the rate of the overlapped area was nearly at a maximum, it was reported by the subjects that the fusion of the two images was easier. On the other hand, the completion time of the task became longer when the convergence point of the stereoscopic cameras was set at a plane farther from the plane of the target object. Fusion became unstable when the rate of the overlapped area of the two images around the target object in the operation was not nearly at a maximum.

\section{Summary}

We examined the efficiency of a teleoperation under three kinds of convergence condition of a stereoscopic camera system. (Condition 1): When the convergence point of the cameras follows the point on the gripper on which subjects fixate both their eyes, the ratio of the overlapped area of the two images is nearly always at a maximum. (Condition 2): The convergence point of the two cameras is not set on the gripper but at the center of the working area. (Condition 3): The convergence point of the two cameras is not set on the gripper but at the hole of the hole-base. The larger the difference of distance between the plane of the convergence point and the plane of the subject's fixation point becomes, the more the rate of the overlapped area of the two images decreases. In the experiment, subjects were required to insert a cylinder into a hole using a three-dimensional mouse in a virtual reality space. We measured the completion times and the number of errors of the operation in each condition. The results of the experiment were that the completion times of the operation under a nearly-perfectly overlapped condition were significantly smaller than the ones under the condition of overlapping less than maximum. These results lead to the conclusion that in order to get good performance in a teleoperation the convergence point of the cameras should follow the target object on which human operators fixate both their eyes. 


\section{References}

1) Nagata, S. (1981). Visual Depth Sensitivities of Various Cues for Depth Perception. NHK LAB. NOTE, 226, 1-11.

2) Drascic, D. (1991, September). Skill Acquisition and Task Performance in Teleoperation using Monoscopic and Stereoscopic Video Remote Viewing. Proceedings of the Human Factors Society 35th Annual Meeting, San Francisco, 1367-1371.

3) Matsunaga, K. (1994). Coordination of body movement and space perception. Bit, 8 (Supplement), $57-64$ ! (in Japanese).

4) Matsunaga, K. (1995). Image environment and operating environment of a remote controlled vehicle. Kyushu
Gihou, 17, 43-48 (in Japanese).

5) Matsunaga, K., Nose, Y., Minamoto, M., Shidoji, K., Ebuchi, K., Itoh, D., Inoue, T., Hayami, T., Matsuki, Y., Arikawa, Y., \& Matsubara, K. (1999). A new stereoscopic video camera and monitor system with central high resolution. Proceedings of 10th International Society for Optical Engineering. Stereoscopic Displays and Virtual Reality Systems V, 164-170.

6) Yamamoto, T., Matsunaga, K., Shidoji, K., \& Matsu$\mathrm{ki}, \mathrm{Y} .(2000)$. The effect of the ratio difference of overlapped areas of stereoscopic images on each eye in a teleoperation. Proceedings of 12th International Society for Optical Engineering. Stereoscopic Displays and Virtual Reality Systems VII, 236-243. 